DOI: 10.12957/demetra.2016.22093

\title{
Fatores associados ao estado nutricional em idosos participantes do Estudo "EpiFloripa Idoso"
}

\section{Factors associated with the nutritional status of elderly participants of the study "EpiFloripa Idoso"}

\author{
Susana Cararo Confortin' \\ Bianca Bittencourt ${ }^{\prime}$ \\ Lariane Mortean $\mathrm{Ono}^{2}$ \\ Larissa Pruner Marques \\ Ione Jayce Ceola Schneider' \\ Eleonora d'Orsi'
I Programa de Pós-graduação em Saúde Coletiva. Universidade Federal de Santa Catarina, Programa de Pós-graduação em Saúde Coletiva. Florianópolis, Santa Catarina, Brasil.
2 Programa de Pós-graduação em Ciências Médicas. Universidade Federal de Santa Catarina, Programa de Pós-graduação em Saúde Coletiva. Florianópolis, Santa Catarina, Brasil. \\ Financiamento: Conselho Nacional de \\ Desenvolvimento Científico e Tecnológico (CNPq), \\ processo número 569834/2008-2. \\ Correspondência / Correspondence \\ Susana Cararo Confortin \\ E-mail: Susanaconfortin@gmail.com
}

\section{Resumo}

Objetivo: Verificar associação entre estado nutricional e fatores demográficos, socioeconômicos, estilo de vida e condições de saúde em idosos. Método: Estudo transversal, base populacional, realizado em 2009, com 1.705 idosos de Florianópolis. O estado nutricional foi avaliado pelo índice de massa corporal. Utilizouse regressão logística multinomial. Resultados: A prevalência de baixo peso foi $7,7 \%$ e $9,9 \%$ e excesso de peso, $56,8 \%$ e $44,9 \%$, para mulheres e homens, respectivamente. Nas mulheres, excesso de peso foi associado a ser insuficientemente ativo, três ou mais morbidades e incapacidade nas atividades de vida diária. Nos homens, baixo peso foi associado à ausência de companheiro e não uso de Internet. $\mathrm{O}$ excesso de peso associou-se à menor renda, ser ex-fumante, fumante atual, incapacidade moderada/ grave e pior percepção de saúde. Ainda, ter 80 anos ou mais e renda no 3 o quartil foram associados ao baixo e excesso de peso nos homens. Conclusões: O estado nutricional foi associado, para mulheres, a hábitos de vida modificáveis, doenças crônicas e incapacidades. Para os homens, o estado nutricional foi mais relacionado aos fatores sociodemográficos. Assim, as estratégias para manutenção do estado nutricional de mulheres e homens devem ser diferenciadas.

Palavras-chave: Estado Nutricional. Idoso. Estudos Transversais. Fatores Socioeconômicos. 


\section{Abstract}

Objective: To determine the association between nutritional status and demographic, socioeconomic, lifestyle, and health condition factors in the elderly. Methods: A population-based, cross-sectional study, held in 2009, with 1705 elderly in Florianópolis. Nutritional status was assessed by body mass index. We analyzed the data using multinomial logistic regression. Results: The prevalence of under- and overweight were $7.7 \%$ and $56.8 \%$ in women, respectively, and $9.9 \%$ and $44.9 \%$ in men. In women, being overweight was associated with being insufficiently active, three or more co-morbidities, and disability in daily living activities. In men, underweight was associated with being single, less educated, and lack of Internet use, whereas overweight was associated with lower incomes, being a former smoker, moderate/ severe disability, and poorer health perception. In men 80 years of age or older, income in the third quartile and smoking were associated with low and overweight, respectively. Conclusions: In women, nutritional status was associated with modifiable lifestyle habits, chronic diseases and disabilities. In men, it was related to sociodemographic factors. Thus, strategies to maintain the nutritional status of women and men must be differentiated.

Key words: Nutritional Status. Aged. Cross-Sectional Studies. Socioeconomic Factors.

\section{Introdução}

No cenário mundial de envelhecimento populacional e de transição epidemiológica, um dos desafios para a saúde pública é manter o envelhecimento ativo e saudável. ${ }^{1}$ Dentre as metas pactuadas pela Estratégia Global para Promoção da Alimentação Saudável, Atividade Física e Saúde, ${ }^{2}$ destaca-se a manutenção de uma dieta equilibrada e, consequentemente, de um peso salutar, visto que o estado nutricional do indivíduo tem papel fundamental na modulação das doenças crônicas e é importante indicador de qualidade de vida e morbimortalidade para os idosos. ${ }^{3}$

O envelhecimento tem impacto negativo sobre o estado nutricional, devido à diminuição da eficiência fisiológica nessa fase. As progressivas mudanças incluem diminuição da capacidade funcional, alterações de processos metabólicos com modificações da composição corporal e falta de apetite. ${ }^{3}$

Contudo, múltiplos determinantes estão associados ao estado nutricional de risco (baixo peso e excesso de peso), como os sociodemográficos, o estilo de vida, as relações sociais e as condições de saúde. ${ }^{4-11}$ A literatura relata que o baixo peso, por exemplo, está associado ao sexo masculino,, 5,12 ao aumento da idade, à menor renda, ${ }^{4,12}$ à autopercepção ruim do estado de saúde, histórico 
clínico de dislipidemias, tabagismo e alterações na função cognitiva. ${ }^{5,12-15}$ Enquanto o excesso de peso está associado às comorbidades, incapacidade funcional, entre outros fatores. ${ }^{4,6,7,9-11-16}$

Apesar da relevância do tema, poucos estudos brasileiros e recentes, que avaliam estes determinantes, foram encontrados, levando em conta a estratificação por sexo, que pode exercer diferentes magnitudes nas medidas de associação. Este estudo permitirá maior compreensão sobre a influência do sexo na determinação do perfil nutricional da população idosa em uma capital do Sul do Brasil. Dessa forma, objetiva verificar a associação entre o estado nutricional e fatores demográficos, socioeconômicos, estilo de vida e condições de saúde, estratificados por sexo, em idosos residentes em Florianópolis, Santa Catarina.

\section{Métodos}

\section{Área e população de estudo}

Os dados deste estudo são parte do projeto "EpiFloripa Idoso" (www.epifloripa.ufsc.br), estudo transversal de base populacional e domiciliar, realizado com idosos (60 anos ou mais) residentes no município de Florianópolis, Estado de Santa Catarina, Sul do Brasil, em 2009/2010.

As informações relacionadas ao local, população do estudo e amostragem foram publicadas em outro estudo ${ }^{17}$ e serão apresentadas brevemente. Foi utilizada a estratégia de amostragem em dois estágios. No primeiro, foram sorteados sistematicamente 80 (oito em cada decil de renda) dentre os 420 setores censitários urbanos do município. As unidades do segundo estágio foram os domicílios, sorteados de maneira sistemática. Todos os idosos residentes nos domicílios sorteados foram convidados a participar do estudo. Dentro dos domicílios selecionados, foram entrevistados 1.705 idosos. Consideraram-se perdas as entrevistas não realizadas após quatro tentativas (inclusive no período noturno e no final de semana) e recusas quando as pessoas optaram em não responder ao questionário.

Para a coleta dos dados, foi usado formulário próprio, na forma de entrevistas face a face, utilizando o Personal Digital Assistant (PDA) para registro dos dados. As entrevistadoras foram previamente treinadas para testagem do instrumento, refinamento e calibração (precisão e exatidão) dos testes.

\section{Variável dependente}

O estado nutricional foi verificado por meio do índice de massa corporal para idosos [IMC = massa corporal $(\mathrm{Kg}) /$ estatura $\left.^{2}(\mathrm{~m})\right]$. As medidas de massa corporal e altura, para a classificação do IMC, foram realizadas de acordo com procedimentos padronizados. ${ }^{18} \mathrm{O}$ IMC foi categorizado 
de acordo com o critério da American Academy of Family Physicians, ${ }^{19}$ adotado no Brasil pelo Sistema de Vigilância Alimentar e Nutricional [SISVAN]: ${ }^{0}<22 \mathrm{Kg} / \mathrm{m}^{2}$, baixo peso; 22, $0 \leq \mathrm{IMC} \leq 27 \mathrm{Kg} /$ $\mathrm{m}^{2}$, adequado; $>27 \mathrm{Kg} / \mathrm{m}^{2}$, excesso de peso.

\section{Variáveis independentes}

Demográficas e socioeconômicas: grupo etário (60 a 69 anos, 70 a 79 e 80 ou mais), estado civil (com companheiro - casado; sem companheiro - solteiro/separado/divorciado e viúvo), trabalhava na época da entrevista (não; sim), escolaridade em anos de estudo $(0$ a $4 ; 5$ a $8 ; 9$ a 11 e $\geq 12) \mathrm{e}$ renda familiar per capita estratificada em quartis ( $1^{\circ}$ quartil: $\leq \mathrm{R} \$ 327,50 ; 2^{\circ}$ quartil: de $\mathrm{R} \$ 327,50$ a $\mathrm{R} \$ 700,00 ; 3^{\circ}$ quartil: de $\mathrm{R} \$ 700,00$ a $\mathrm{R} \$ 1.500,00$ e $4^{\underline{0}}$ quartil: > $\left.\mathrm{R} \$ 1.500,00\right)$.

Estilo de vida: consumo de álcool (nunca, moderado, alto ou de risco) verificado por meio das três primeiras perguntas do questionário AUDIT (The Alcohol Use Disorders IdentificationTest), ${ }^{21}$ tabagismo (fumante atual, ex-fumante e nunca fumou), atividade física de lazer verificada através do Questionário Internacional de Atividade Física (IPAQ), ${ }^{22}$ versão longa ( 0 a $<10$ minutos; de 10 a 149 minutos e $\geq 150$ minutos de atividades físicas/semana).

Uso de Internet: capacidade autorreferida em usar computador para envio e recebimento de mensagens pela Internet. ${ }^{23}$

Condições de saúde: número de morbidades (nenhuma a duas morbidades, três ou mais morbidades relatadas) verificado a partir do questionamento "Algum médico ou profissional de saúde já disse que o(a) Sr.(a) tem/teve...?”, no qual constavam 16 opções de agravos (questionário da Pesquisa Nacional por Amostra de Domicílios - PNAD), ${ }^{24} \mathrm{e}$ incapacidade funciona ${ }^{25}$ (ausência de dependência, dependência leve [dependência em uma a três atividades] e moderada/grave [dependência em quatro ou mais atividades]) avaliada por meio do Questionário Brasileiro de Avaliação Funcional Multidimensional adaptado do questionário Old Americans Resources and Services (BOMFAQ/OARS). ${ }^{25}$ A autopercepção do estado de saúde foi obtida por meio do questionamento "Em geral, você diria que sua saúde é: muito boa, boa, regular, ruim ou muito ruim?". ${ }^{26}$ As cinco categorias de resposta para o desfecho foram categorizadas em "muito boa/boa", "regular" e "ruim/muito ruim").

\section{Análise dos dados}

Foram realizadas análises descritivas para todas as variáveis. Calcularam-se as prevalências e os respectivos intervalos de confiança (IC95\%) do estado nutricional segundo a natureza das exposições. Para a análise bruta e ajustada, foi utilizada regressão logística multinomial, estimandose as Razões de Odds (OR) com seus respectivos intervalos de confiança de 95\% (IC95\%). Para a 
análise ajustada, fez-se uso, no modelo final, de todas as variáveis: demográficas, socioeconômicas, uso de Internet e as condições de saúde. Todas as análises foram estratificadas por sexo. Considerouse o nível de significância estatística de $5 \%$.

O programa estatístico Stata SE 13.0 (StataCorp.,College Station, EUA) foi empregado para conduzir a análise dos dados. Todas as análises realizadas consideram o efeito do desenho amostral por conglomerados com a incorporação dos pesos amostrais.

O protocolo de estudo foi aprovado pelo Comitê de Ética em Pesquisa com Seres Humanos da Universidade Federal de Santa Catarina (nº 352/2008). A participação foi voluntária, com a obtenção de um termo de consentimento livre e esclarecido. Os autores declaram não haver conflito de interesses.

\section{Resultados}

Do total de idosos elegíveis para o estudo $(n=1911), 1.705$ foram entrevistados, com taxa de resposta de $89,1 \%$. O estado nutricional foi avaliado em 1.646 idosos, pois 59 participantes não tinham informações suficientes para o cálculo do IMC. Observou-se que 63,9\% eram do sexo feminino.

A maior frequência de mulheres idosas foi no grupo de 60 a 69 anos e apresentava de zero a quatro anos de estudo (46,0\%). Mais da metade relatou não ter companheiro $(55,2 \%)$ e não trabalhar no momento da entrevista (90,1\%). Mais de 70,0\% não consumia bebidas alcoólicas, reportou nunca ter fumado, e menos de 1/5 das idosas praticava 150 minutos por semana de atividade física no lazer (16,2\%). Grande parte relatou não utilizar a Internet (83,2\%), ter três ou mais morbidades $(59,9 \%)$ e incapacidade leve nas atividades de vida diária (AVD) (39,3\%) (Tabela 1).

Tabela 1. Descrição da amostra e análise bivariada, de acordo com as variáveis demográficas, socioeconômicas, estilo de vida, uso de Internet e condições de saúde em mulheres e homens de Florianópolis, Santa Catarina, 2009/2010.

\begin{tabular}{|c|c|c|c|c|c|c|c|c|}
\hline \multirow[b]{2}{*}{ Variáveis } & \multicolumn{4}{|c|}{ Mulheres } & \multicolumn{4}{|c|}{ Homens } \\
\hline & $\mathrm{n}$ & $\begin{array}{c}\% \text { Baixo } \\
\text { peso }\end{array}$ & $\begin{array}{c}\text { \% Excesso } \\
\text { de peso }\end{array}$ & Valor de $\mathrm{p}$ & $\mathrm{n}$ & $\begin{array}{c}\% \text { Baixo } \\
\text { peso }\end{array}$ & $\begin{array}{c}\% \text { Excesso } \\
\text { de peso }\end{array}$ & Valor de $\mathrm{p}$ \\
\hline Grupo etário & & & & 0,175 & & & & $\leq 0,001$ \\
\hline 60 a 69 anos & 530 & 8,05 & 58,43 & & 324 & 6,29 & 49,37 & \\
\hline 70 a 79 anos & 400 & 5,94 & 57,11 & & 212 & 10,84 & 42,86 & \\
\hline 80 anos ou mais & 159 & 11,19 & 50,35 & & 80 & 23,29 & 31,51 & \\
\hline Total & 1089 & & & & 616 & & & \\
\hline
\end{tabular}




\begin{tabular}{|c|c|c|c|c|c|c|c|c|}
\hline \multirow[b]{2}{*}{ Variáveis } & \multicolumn{4}{|c|}{ Mulheres } & \multicolumn{4}{|c|}{ Homens } \\
\hline & $\mathrm{n}$ & $\begin{array}{c}\% \text { Baixo } \\
\text { peso }\end{array}$ & $\begin{array}{c}\% \text { Excesso } \\
\text { de peso }\end{array}$ & Valor de $\mathrm{p}$ & $\mathrm{n}$ & $\begin{array}{c}\% \text { Baixo } \\
\text { peso }\end{array}$ & $\begin{array}{c}\% \text { Excesso } \\
\text { de peso }\end{array}$ & Valor de $\mathrm{p}$ \\
\hline Estado civil & & & & 0,388 & & & & 0,120 \\
\hline Com companheiro(a) & 488 & 6,93 & 59,03 & & 505 & 8,78 & 45,71 & \\
\hline Sem companheiro(a) & 601 & 8,33 & 55,03 & & 111 & 15,38 & 41,35 & \\
\hline Total & 1089 & & & & 616 & & & \\
\hline Trabalha atualmente & & & & 0,526 & & & & 0,054 \\
\hline Sim & 108 & 10,28 & 57,01 & & 121 & 5,00 & 53,50 & \\
\hline Não & 981 & 7,41 & 56,83 & & 495 & 11,18 & 43,04 & \\
\hline Total & 1089 & & & & 616 & & & \\
\hline Escolaridade & & & & 0,408 & & & & 0,004 \\
\hline$\geq 12$ anos & 202 & 6,57 & 52,53 & & 192 & 3,76 & 54,84 & \\
\hline 9 a 11 anos & 158 & 7,10 & 62,58 & & 76 & 8,00 & 44,00 & \\
\hline 5 a 8 anos & 244 & 7,91 & 54,42 & & 97 & 13,98 & 39,78 & \\
\hline 0 a 4 anos & 497 & 8,35 & 58,04 & & 248 & 13,87 & 39,92 & \\
\hline Total & 1101 & & & & 613 & & & \\
\hline \multicolumn{2}{|c|}{ Renda per capita em reais } & & & 0,372 & & & & $\leq 0,001$ \\
\hline $4^{\circ}$ quartil & 246 & 5,83 & 53,33 & & 172 & 6,59 & 56,89 & \\
\hline $3^{\circ}$ quartil & 259 & 6,88 & 57,09 & & 166 & 4,32 & 45,06 & \\
\hline $2^{\circ}$ quartil & 284 & 8,42 & 59,71 & & 151 & 16,67 & 39,58 & \\
\hline $1^{o}$ quartil (menor) & 300 & 9,25 & 56,85 & & 127 & 14,05 & 34,71 & \\
\hline Total & 1089 & & & & 616 & & & \\
\hline Consumo de álcool & & & & 0,682 & & & & 0,027 \\
\hline Não consome & 816 & 8,16 & 56,25 & & 292 & 12,00 & 38,55 & \\
\hline Moderado & 190 & 7,53 & 57,53 & & 126 & 10,40 & 46,40 & \\
\hline Alto & 83 & 3,66 & 60,98 & & 198 & 6,70 & 53,09 & \\
\hline Total & 1089 & & & & 616 & & & \\
\hline Tabagismo & & & & 0,222 & & & & $\leq 0,001$ \\
\hline Nunca fumou & 814 & 7,53 & 56,25 & & 225 & 7,83 & 54,38 & \\
\hline Ex-fumante & 208 & 6,83 & 62,44 & & 315 & 9,27 & 42,72 & \\
\hline
\end{tabular}




\begin{tabular}{|c|c|c|c|c|c|c|c|c|}
\hline \multirow[b]{2}{*}{ Variáveis } & \multicolumn{4}{|c|}{ Mulheres } & \multicolumn{4}{|c|}{ Homens } \\
\hline & $\mathrm{n}$ & $\begin{array}{c}\% \text { Baixo } \\
\text { peso }\end{array}$ & $\begin{array}{c}\text { \% Excesso } \\
\text { de peso }\end{array}$ & Valor de $\mathrm{p}$ & $\mathrm{n}$ & $\begin{array}{c}\% \text { Baixo } \\
\text { peso }\end{array}$ & $\begin{array}{c}\text { \% Excesso } \\
\text { de peso }\end{array}$ & Valor de $\mathrm{p}$ \\
\hline Fumante atual & 65 & 11,29 & 46,77 & & 76 & 18,67 & 26,67 & \\
\hline Total & 1087 & & & & 616 & & & \\
\hline Atividade física no lazer & & & & 0,086 & & & & 0,519 \\
\hline 150 minutos ou mais & 176 & 10,29 & 48,00 & & 147 & 11,11 & 43,06 & \\
\hline 10 a 149 minutos & 157 & 5,13 & 60,26 & & 97 & 5,26 & 45,26 & \\
\hline 0 a $<10$ minutos & 756 & 7,63 & 58,25 & & 372 & 10,70 & 45,63 & \\
\hline Total & 1089 & & & & 616 & & & \\
\hline Uso de Internet & & & & 0,172 & & & & $<0,001$ \\
\hline Sim & 182 & 6,74 & 51,89 & & 163 & 1,86 & 52,17 & \\
\hline Não & 907 & 7,89 & 57,89 & & 453 & 12,93 & 42,26 & \\
\hline Total & 1089 & & & & 616 & & & \\
\hline Número de morbidades & & & & $<0,001$ & & & & 0,076 \\
\hline 0 a 2 morbidades & 431 & 9,0 & 46,45 & & 346 & 10,36 & 40,83 & \\
\hline 3 ou mais morbidades & 643 & 6,98 & 63,96 & & 265 & 9,16 & 50,20 & \\
\hline Total & 1074 & & & & 611 & & & \\
\hline Incapacidade nas AVD & & & & $<0,001$ & & & & 0,433 \\
\hline $\begin{array}{l}\text { Ausência de } \\
\text { incapacidade }\end{array}$ & 275 & 7,66 & 46,72 & & 183 & 8,24 & 42,86 & \\
\hline Leve & 431 & 7,96 & 57,85 & & 278 & 9,35 & 45,68 & \\
\hline Moderada/grave & 383 & 7,41 & 63,53 & & 155 & 13,43 & 46,27 & \\
\hline Total & 1089 & & & & 616 & & & \\
\hline Autopercepção do estado & de saúd & & & 0,082 & & & & 0,279 \\
\hline Muito boa/boa & 509 & 8,03 & 52,41 & & 351 & 8,93 & 44,96 & \\
\hline Regular & 438 & 7,44 & 60,70 & & 208 & 11,33 & 47,78 & \\
\hline Ruim/muito ruim & 124 & 6,72 & 62,18 & & 51 & 9,30 & 32,56 & \\
\hline Total & 1071 & & & & 610 & & & \\
\hline
\end{tabular}


Quanto à população masculina, a maior frequência de idosos foi no grupo etário de 60 a 69 anos (52,6\%). Mais da metade relatou ter companheiro(a) $(82,0 \%)$ e não trabalhava no momento da entrevista $(80,4 \%)$. A maior prevalência foi de idosos de zero a quatro anos de estudo $(40,5 \%)$, que não consomem álcool (47,4\%), ex-fumantes $(51,1 \%)$, insuficientemente ativo no lazer $(60,4 \%)$, relatou não utilizar Internet $(73,5 \%)$, nenhuma a duas morbidades $(56,6 \%)$, incapacidade leve nas AVD $(45,13 \%)$ e autopercepção muito boa/boa do estado de saúde $(57,5 \%)$ (Tabela 1$)$.

A prevalência de baixo peso foi de 8,5\% (IC95\%: 0,07-0,10) e excesso de peso de 52,5\% (IC95\%: 50,10-54,96). A proporção de mulheres com baixo peso foi 7,7\% (IC95\%: 6,23-9,48) e excesso de peso, 56,8\% (IC95\%: 53,82-59,81). Enquanto nos homens, 9,9\% (IC95\%: 7,77-12,62) apresentaram baixo peso e 44,9\% (IC95\%: 40,98-48,99), excesso de peso.

Os resultados das associações, na análise bruta e ajustada, entre o estado nutricional e variáveis independentes, em mulheres e homens, estão indicados na Tabela 2 e na Tabela 3, respectivamente.

Tabela 2. Análise bruta e ajustada para mulheres, em relação aos fatores associados ao estado nutricional. Florianópolis, Santa Catarina, Brasil, 2009/2010.

\begin{tabular}{|c|c|c|c|c|c|c|}
\hline \multirow{3}{*}{ Variáveis } & \multicolumn{3}{|c|}{ Baixo Peso } & \multicolumn{3}{|c|}{ Excesso de Peso } \\
\hline & \multicolumn{2}{|c|}{$\begin{array}{c}\text { Análise } \\
\text { bruta }\end{array}$} & \multirow{2}{*}{$\begin{array}{c}\begin{array}{c}\text { Análise } \\
\text { ajustada }\end{array} \\
\text { OR (IC95\%) }\end{array}$} & \multicolumn{2}{|c|}{$\begin{array}{c}\text { Análise } \\
\text { bruta }\end{array}$} & \multirow{2}{*}{$\begin{array}{c}\text { Análise ajustada } \\
\text { OR (IC95\%) }\end{array}$} \\
\hline & OR (IC95\%) & $\begin{array}{l}\text { Valor } \\
\text { de p }\end{array}$ & & OR (IC95\%) & $\begin{array}{l}\text { Valor } \\
\text { de } p\end{array}$ & \\
\hline Grupo etário $(\mathrm{n}=1052)$ & & 0,471 & & & 0,309 & \\
\hline 60 a 69 anos & 1 & & 1 & 1 & & 1 \\
\hline 70 a 79 anos & $0,74(0,36-1,51)$ & & $0,65(0,32-1,32)$ & $0,91(0,61-1,36)$ & & $0,75(0,48-1,16)$ \\
\hline 80 anos ou mais & $1,77(0,63-4,90)$ & & $1,82(0,63-5,28)$ & $0,76(0,49-1,19)$ & & $0,68(0,41-1,14)$ \\
\hline Estado civil $(\mathrm{n}=1052)$ & & 0,348 & & & 0,610 & \\
\hline Com companheiro & 1 & & - & 1 & & - \\
\hline Sem companheiro & $1,26(0,77-2,08)$ & & - & $0,94(0,68-1,26)$ & & - \\
\hline \multicolumn{2}{|c|}{ Trabalha atualmente $(\mathrm{n}=1052)$} & 0,139 & & & 0,290 & \\
\hline Não & 1 & & - & 1 & & - \\
\hline Sim & $0,60(0,30-1,19)$ & & - & $0,73(0,40-1,32)$ & & - \\
\hline
\end{tabular}




\begin{tabular}{|c|c|c|c|c|c|c|}
\hline \multirow{4}{*}{ Variáveis } & \multicolumn{3}{|c|}{ Baixo Peso } & \multicolumn{3}{|c|}{ Excesso de Peso } \\
\hline & \multirow{2}{*}{\multicolumn{2}{|c|}{$\begin{array}{c}\text { Análise } \\
\text { bruta }\end{array}$}} & \multirow{3}{*}{$\begin{array}{c}\begin{array}{c}\text { Análise } \\
\text { ajustada }\end{array} \\
\text { OR (IC95\%) }\end{array}$} & \multirow{2}{*}{\multicolumn{2}{|c|}{$\begin{array}{c}\text { Análise } \\
\text { bruta }\end{array}$}} & \multirow{3}{*}{$\begin{array}{l}\text { Análise ajustada } \\
\text { OR (IC95\%) }\end{array}$} \\
\hline & & & & & & \\
\hline & OR (IC95\%) & $\begin{array}{l}\text { Valor } \\
\text { de p }\end{array}$ & & OR (IC95\%) & $\begin{array}{l}\text { Valor } \\
\text { de p }\end{array}$ & \\
\hline Escolaridade $(n=1047)$ & & 0,394 & & & 0,229 & \\
\hline$\geq 12$ anos & 1 & & - & 1 & & - \\
\hline 9 a 11 anos & $1,37(0,53-3,56)$ & & - & $1,52(0,90-2,59)$ & & - \\
\hline 5 a 8 anos & $1,17(0,52-2,66)$ & & - & $1,01(0,60-1,72)$ & & - \\
\hline 0 a 4 anos & $1,51(0,63-3,62)$ & & - & $1,42(0,94-2,13)$ & & - \\
\hline \multicolumn{2}{|c|}{ Renda per capita em reais $(n=1052)$} & 0,105 & & & 0,625 & \\
\hline $4^{\circ}$ quartil & 1 & & 1 & 1 & & 1 \\
\hline $3^{o}$ quartil & $1,88(0,88-4,03)$ & & $1,83(0,84-3,98)$ & $1,15(0,85-1,57)$ & & $1,14(0,84-1,13)$ \\
\hline $2^{\circ}$ quartil & $2,15(0,92-5,03)$ & & $1,95(0,79-4,78)$ & $1,27(0,77-2,08)$ & & $1,03(0,61-1,72)$ \\
\hline $1^{\mathrm{o}}$ quartil (menor) & $2,23(0,96-5,17)$ & & $2,21(0,91-5,36)$ & $1,09(0,70-1,70)$ & & $1,02(0,66-1,57)$ \\
\hline \multicolumn{2}{|l|}{ Consumo de álcool (n=1052) } & 0,172 & & & 0,131 & \\
\hline Não consome & 1 & & 1 & 1 & & 1 \\
\hline Moderado & $0,84(0,41-1,72)$ & & $1,05(0,52-2,14)$ & $1,17(0,78-1,75)$ & & $1,33(0,87-2,03)$ \\
\hline Alto & $0,40(0,08-1,93)$ & & $0,51(0,11-2,14)$ & $1,39(0,79-2,46)$ & & $1,72(0,98-3,03)$ \\
\hline Tabagismo $(n=1051)$ & & 0,952 & & & 0,955 & \\
\hline Nunca fumou & 1 & & - & 1 & & - \\
\hline Ex-fumante & $1,09(0,52-2,25)$ & & - & $1,42(0,92-2,20)$ & & - \\
\hline Fumante atual & $0,97(0,31-3,00)$ & & - & $0,67(0,30-1,48)$ & & - \\
\hline Atividade física no lazer $(\mathrm{n}=$ & $=1052)$ & 0,777 & & & 0,078 & \\
\hline 150 minutos ou mais & 1 & & 1 & 1 & & 1 \\
\hline 10 a 149 minutos & $0,62(0,21-1,85)$ & & $0,53(0,18-1,60)$ & $1,83(1,05-3,22)$ & & $1,74(1,03-2,91)$ \\
\hline $0 \mathrm{a}<10$ minutos & $0,90(0,45-1,67)$ & & $0,70(0,34-1,45)$ & $1,56(1,05-2,33)$ & & $1,35(0,92-1,99)$ \\
\hline Uso de Internet ( $n=1052)$ & & 0,378 & & & 0,238 & \\
\hline Sim & 1 & & - & 1 & & - \\
\hline Não & $1,56(0,58-4,21)$ & & - & $1,39(0,80-2,43)$ & & - \\
\hline
\end{tabular}




\begin{tabular}{|c|c|c|c|c|c|c|}
\hline \multirow{3}{*}{ Variáveis } & \multicolumn{3}{|c|}{ Baixo Peso } & \multicolumn{3}{|c|}{ Excesso de Peso } \\
\hline & \multicolumn{2}{|c|}{$\begin{array}{c}\text { Análise } \\
\text { bruta }\end{array}$} & \multirow{2}{*}{$\begin{array}{c}\begin{array}{c}\text { Análise } \\
\text { ajustada }\end{array} \\
\text { OR (IC95\%) }\end{array}$} & \multicolumn{2}{|l|}{$\begin{array}{c}\text { Análise } \\
\text { bruta }\end{array}$} & \multirow{2}{*}{$\begin{array}{l}\text { Análise ajustada } \\
\text { OR (IC95\%) }\end{array}$} \\
\hline & OR (IC95\%) & $\begin{array}{l}\text { Valor } \\
\text { de p }\end{array}$ & & OR (IC95\%) & $\begin{array}{l}\text { Valor } \\
\text { de p }\end{array}$ & \\
\hline \multicolumn{2}{|c|}{ Número de morbidades $(n=1038)$} & 0,502 & & & 0,000 & \\
\hline 0 a 2 morbidades & 1 & & 1 & 1 & & 1 \\
\hline 3 ou mais morbidades & $1,39(0,86-2,26)$ & & $1,28(0,72-2,25)$ & $0,39(1,58-3,15)$ & & $1,86(1,29-2,69)$ \\
\hline \multicolumn{2}{|c|}{ Incapacidade nas AVD $(n=1052)$} & 0,216 & & & 0,000 & \\
\hline Ausência & 1 & & 1 & 1 & & 1 \\
\hline Leve & $1,46(0,75-2,84)$ & & $1,31(0,64-2,68)$ & $1,60(1,13-2,27)$ & & $1,59(1,11-2,27)$ \\
\hline Moderada/grave & $1,75(0,72-4,24)$ & & $1,24(0,44-3,48)$ & $2,22(1,44-3,42)$ & & $1,94(1,15-3,27)$ \\
\hline \multicolumn{2}{|c|}{ Autopercepção do estado de saúde $(n=1047)$} & 0,145 & & & 0,005 & \\
\hline Muito boa/boa & 1 & & 1 & 1 & & 1 \\
\hline Regular & $1,74(0,88-3,43)$ & & $1,47(0,70-3,07)$ & $1,64(1,22-2,21)$ & & $1,28(0,91-1,77)$ \\
\hline Ruim/muito ruim & $1,38(0,54-3,52)$ & & $0,96(0,36-2,51)$ & $1,61(0,92-2,80)$ & & $0,98(0,53-1,79)$ \\
\hline
\end{tabular}

Tabela 3. Análise bruta e ajustada para homens, em relação aos fatores associados ao estado nutricional. Florianópolis, Santa Catarina, Brasil, 2009/2010.

\begin{tabular}{|c|c|c|c|c|c|c|}
\hline \multirow{3}{*}{ Variáveis } & \multicolumn{3}{|c|}{ Baixo peso } & \multicolumn{3}{|c|}{ Excesso de Peso } \\
\hline & \multicolumn{2}{|l|}{$\begin{array}{c}\text { Análise } \\
\text { bruta }\end{array}$} & \multirow{2}{*}{$\begin{array}{c}\begin{array}{c}\text { Análise } \\
\text { ajustada }\end{array} \\
\text { OR (IC95\%) }\end{array}$} & \multicolumn{2}{|l|}{$\begin{array}{c}\text { Análise } \\
\text { bruta }\end{array}$} & \multirow{2}{*}{$\begin{array}{c}\begin{array}{c}\text { Análise } \\
\text { ajustada }\end{array} \\
\text { OR (IC95\%) }\end{array}$} \\
\hline & OR (IC95\%) & $\begin{array}{l}\text { Valor } \\
\text { de p }\end{array}$ & & OR (IC95\%) & $\begin{array}{l}\text { Valor } \\
\text { de } \mathrm{p}\end{array}$ & \\
\hline Grupo etário $(\mathrm{n}=594)$ & & $<0,001$ & & & 0,264 & \\
\hline 60 a 69 anos & 1 & & 1 & 1 & & 1 \\
\hline 70 a 79 anos & $2,48(0,95-6,50)$ & & $1,74(0,54-5,58)$ & $1,02(0,67-1,55)$ & & $0,78(0,48-1,28)$ \\
\hline 80 anos ou mais & $5,68(2,89-11,52)$ & & $6,35(1,87-21,60)$ & $0,57(0,29-1,12)$ & & $0,32(0,13-0,74)$ \\
\hline Estado civil $(n=594)$ & & 0,015 & & & 0,569 & \\
\hline Com companheiro & 1 & & 1 & 1 & & 1 \\
\hline Sem companheiro & $2,57(1,34-4,92)$ & & 2,73(1,21-6,15) & $0,88(0,56-1,37)$ & & $0,82(0,48-1,39)$ \\
\hline
\end{tabular}




\begin{tabular}{|c|c|c|c|c|c|c|}
\hline \multirow{3}{*}{ Variáveis } & \multicolumn{3}{|c|}{ Baixo peso } & \multicolumn{3}{|c|}{ Excesso de Peso } \\
\hline & \multicolumn{2}{|l|}{$\begin{array}{c}\text { Análise } \\
\text { bruta }\end{array}$} & \multirow{2}{*}{$\begin{array}{c}\begin{array}{c}\text { Análise } \\
\text { ajustada }\end{array} \\
\text { OR (IC95\%) }\end{array}$} & \multicolumn{2}{|l|}{$\begin{array}{c}\text { Análise } \\
\text { bruta }\end{array}$} & \multirow{2}{*}{$\begin{array}{c}\begin{array}{c}\text { Análise } \\
\text { ajustada }\end{array} \\
\text { OR (IC95\%) }\end{array}$} \\
\hline & OR (IC95\%) & $\begin{array}{l}\text { Valor } \\
\text { de p }\end{array}$ & & OR (IC95\%) & $\begin{array}{l}\text { Valor } \\
\text { de p }\end{array}$ & \\
\hline \multicolumn{2}{|l|}{ Trabalha atualmente $(\mathrm{n}=594)$} & 0,219 & & & 0,343 & \\
\hline Não & 1 & & - & 1 & & - \\
\hline Sim & $2,24(0,61-8,20)$ & & - & $0,72(0,36-1,43)$ & & - \\
\hline Escolaridade $(\mathrm{n}=592)$ & & $<0,001$ & & & 0,069 & \\
\hline$\geq 12$ anos & 1 & & 1 & 1 & & 1 \\
\hline 9 a 11 anos & $1,57(0,41-6,03)$ & & $0,90(0,17-4,76)$ & $0,41(0,23-0,74)$ & & $0,59(0,29-1,20)$ \\
\hline 5 a 8 anos & $4,54(1,42-14,48)$ & & $2,86(0,78-10,50)$ & $0,57(0,25-1,26)$ & & $0,71(0,29-1,20)$ \\
\hline 0 a 4 anos & $4,56(2,08-10,01)$ & & $1,65(0,47-5,84)$ & $0,62(0,38-0,99)$ & & $0,92(0,42-2,01)$ \\
\hline \multicolumn{2}{|c|}{ Renda per capita em reais $(\mathrm{n}=594)$} & 0,013 & & & 0,000 & \\
\hline $4^{\circ}$ quartil & 1 & & 1 & 1 & & 1 \\
\hline $3^{\circ}$ quartil & $0,37(0,13-0,97)$ & & $0,12(0,03-0,46)$ & $0,47(0,28-0,80)$ & & $0,48(0,24-0,96)$ \\
\hline $2^{\circ}$ quartil & $2,48(0,96-6,39)$ & & $1,02(0,34-3,05)$ & $0,46(0,28-0,77)$ & & $0,41(0,19-0,88)$ \\
\hline 1º quartil (menor) & $2,59(0,91-7,36)$ & & $0,79(0,24-2,61)$ & $0,37(0,23-0,57)$ & & $0,31(0,15-0,66)$ \\
\hline Consumo de álcool $(n=594)$ & & 0,066 & & & 0,066 & \\
\hline Não consome & 1 & & 1 & 1 & & 1 \\
\hline Moderado & $0,73(0,25-2,14)$ & & $1,57(0,57-4,33)$ & $1,21(0,83-1,78)$ & & $1,27(0,84-1,90)$ \\
\hline Alto & $0,41(0,17-0,98)$ & & $0,72(0,30-1,75)$ & $1,53(0,96-2,42)$ & & $1,52(0,98-2,33)$ \\
\hline Tabagismo $(\mathrm{n}=594)$ & & 0,547 & & & 0,004 & \\
\hline Nunca fumou & 1 & & 1 & 1 & & 1 \\
\hline Ex-fumante & $0,77(0,31-1,91)$ & & $0,63(0,24-1,66)$ & $0,65(0,45-0,94)$ & & $0,59(0,37-0,94)$ \\
\hline Fumante atual & $1,58(0,59-4,26)$ & & $2,08(0,65-6,66)$ & $0,39(0,18-0,87)$ & & $0,30(0,12-0,73)$ \\
\hline Atividade física no lazer $(n=59$ & 94) & 0,974 & & & 0,197 & \\
\hline 150 minutos ou mais & 1 & & 1 & 1 & & 1 \\
\hline 10 a 149 minutos & $0,81(0,13-4,97)$ & & $0,70(0,11-4,36)$ & $1,55(0,67-3,57)$ & & $1,55(0,75-3,21)$ \\
\hline $0 \mathrm{a}<10$ minutos & $0,97(0,40-2,32)$ & & $0,57(0,20-1,64)$ & $1,57(0,80-3,05)$ & & $1,67(0,89-3,11)$ \\
\hline
\end{tabular}




\begin{tabular}{|c|c|c|c|c|c|c|}
\hline \multirow{3}{*}{ Variáveis } & \multicolumn{3}{|c|}{ Baixo peso } & \multicolumn{3}{|c|}{ Excesso de Peso } \\
\hline & \multicolumn{2}{|l|}{$\begin{array}{c}\text { Análise } \\
\text { bruta }\end{array}$} & \multirow{2}{*}{$\begin{array}{c}\begin{array}{c}\text { Análise } \\
\text { ajustada }\end{array} \\
\text { OR (IC95\%) }\end{array}$} & \multicolumn{2}{|l|}{$\begin{array}{c}\text { Análise } \\
\text { bruta }\end{array}$} & \multirow{2}{*}{$\begin{array}{c}\begin{array}{c}\text { Análise } \\
\text { ajustada }\end{array} \\
\text { OR }(\mathrm{IC} 95 \%)\end{array}$} \\
\hline & OR (IC95\%) & $\begin{array}{l}\text { Valor } \\
\text { de p }\end{array}$ & & OR (IC95\%) & $\begin{array}{l}\text { Valor } \\
\text { de p }\end{array}$ & \\
\hline Uso de Internet $(n=594)$ & & $<0,001$ & & & 0,622 & \\
\hline $\operatorname{Sim}$ & 1 & & 1 & 1 & & 1 \\
\hline Não & $14,78(4,27-51,03)$ & & $10,62(1,83-61,78)$ & $0,90(0,60-1,36)$ & & $1,77(0,96-3,29)$ \\
\hline \multicolumn{2}{|c|}{ Número de morbidades $(n=589)$} & 0,775 & & & 0,090 & \\
\hline 0 a 2 morbidades & 1 & & - & 1 & & - \\
\hline 3 ou mais morbidades & $1,13(0,48-2,64)$ & & - & $1,52(0,93-2,46)$ & & - \\
\hline \multicolumn{2}{|c|}{ Incapacidade nas AVD $(n=594)$} & 0,044 & & & 0,073 & \\
\hline Ausência & 1 & & 1 & 1 & & 1 \\
\hline Leve & $1,38(0,63-3,05)$ & & $1,30(0,49-3,47)$ & $1,32(0,80-2,17)$ & & $1,33(0,79-2,25)$ \\
\hline Moderada/grave & $2,42(1,07-5,48)$ & & $0,89(0,18-4,50)$ & $1,76(0,95-3,25)$ & & $3,37(1,65-6,87)$ \\
\hline \multicolumn{2}{|c|}{ Autopercepção do estado de saúde $(n=593)$} & 0,500 & & & 0,442 & \\
\hline Muito boa/boa & 1 & & 1 & 1 & & 1 \\
\hline Regular & $1,30(0,59-2,84)$ & & $1,26(0,51-3,13)$ & $1,28(0,81-2,04)$ & & $0,95(0,64-1,41)$ \\
\hline Ruim/muito ruim & $1,29(0,38-4,40)$ & & $0,42(0,10-1,71)$ & $0,38(0,17-0,84)$ & & $0,30(0,64-0,70)$ \\
\hline
\end{tabular}

Para as mulheres, na análise ajustada, nenhuma variável independente apresentou associação significativa com o baixo peso. No que se refere ao excesso de peso, a análise ajustada mostra que a probabilidade foi 1,74 vez maior nas mulheres insuficientemente ativas no lazer quando comparadas às suficientemente ativas. Observou-se, também, que a probabilidade foi 1,86 vez maior nas mulheres com três ou mais morbidades comparadas aos seus pares. Além disso, houve aumento da probabilidade do desfecho em $59 \%$ e $94 \%$ naquelas com incapacidade leve e moderada nas AVD, respectivamente, comparadas às sem incapacidade (Tabela 2).

Para o sexo masculino, na análise ajustada, a probabilidade de baixo peso foi 6,35 vezes maior nos idosos com 80 anos ou mais e 2,73 vezes maior nos sem companheiro quando comparados aos seus pares. Os idosos que não usam Internet apresentaram 10,62 vezes maior probabilidade de baixo peso quando comparados aos que usam Internet. Já os que estavam no 3o quartil de renda, 0,12 vez a probabilidade de baixo peso. No que se refere ao excesso de peso, os homens com incapacidade moderada/grave em AVD apontaram probabilidade 3,37 maior de apresentar 
o desfecho, quando comparados aos seus pares. Observou-se, também, que a probabilidade de excesso de peso foi $68 \%$ menor nos idosos com 80 anos ou mais, $52 \%$ menor nos com renda no terceiro quartil, $59 \%$ menor nos com renda no segundo quartil e $69 \%$ menor nos com renda no primeiro quartil. Em relação ao hábito tabágico, a probabilidade de excesso de peso é $41 \%$ e $70 \%$ menor nos ex-fumantes e fumantes atuais, respectivamente, quando comparados aos seus pares. Assim como os idosos com autopercepção ruim/muito ruim do estado de saúde apresentaram 30\% menos probabilidade de excesso de peso.

\section{Discussão}

\section{Sexo feminino}

O excesso de peso foi associado à prática reduzida de atividade física para as mulheres, que, normalmente, praticam menos exercícios quando comparadas aos homens, ${ }^{27}$ o que acarreta menor gasto energético, ${ }^{28}$ podendo repercutir em excesso de peso. Adicionado à menor prática de atividade física das mulheres, essas tendem a acumular mais gordura durante a menopausa, com distribuição central, o que seria agravado pela falta de atividade física. ${ }^{29}$ Os resultados de Meneguci et al. ${ }^{30}$ vão de encontro aos do presente estudo, no qual houve associação do aumento de peso com a presença de doenças crônicas, como hipertensão e diabetes mellitus. Machado et al. ${ }^{31}$ reforçam a hipótese de que a presença dessas enfermidades reflete o aumento do IMC, quando observaram que a probabilidade de multimorbidade aumenta 3\% a cada aumento de 1 ponto no IMC. A obesidade pode ser a consequência da presença de doenças crônicas e também pode estar associada à inflamação crônica, que leva ao aumento de doenças recorrentes. A relação com excesso de peso pode predispor o desenvolvimento de outras comorbidades ${ }^{32}$ e aumentar o risco de mortalidade, gerando, consequentemente, impacto sobre o sistema de saúde. ${ }^{4,31}$

No que diz respeito à incapacidade funcional, nas mulheres, o excesso de peso apresentou associação com incapacidade leve e moderada/grave para realização de AVD, assim como o achado por Lisko et al. ${ }^{6}$ A obesidade é associada ao aumento de incapacidade entre idosas por afetar a mobilidade. ${ }^{9}$ Barbosa et al. ${ }^{7}$ sugerem que a presença de incapacidade pode reduzir o nível de atividade física realizada e, consequentemente, aumentar o peso corporal.

\section{Sexo Masculino}

No presente estudo foi encontrada associação positiva entre baixo peso, e negativa com excesso de peso, em idosos do sexo masculino com 80 anos ou mais, assim como verificado por outros autores. ${ }^{4,5,12}$ Essa associação pode ser decorrente da suscetibilidade das deficiências nutricionais e do maior número de problemas de saúde que homens idosos podem apresentar. ${ }^{12}$ No presente 
estudo foi encontrada redução do excesso de peso com diminuição do quartil de renda. O baixo peso apresentou redução somente no terceiro quartil de renda. Campos et al. ${ }^{4}$ observaram aumento do excesso de peso relacionado com o da renda, e renda entre 2 e 5 salários mínimos associada ao baixo peso. Monteiro et al. ${ }^{33}$ notaram que o aumento da renda familiar eleva o risco de obesidade em homens. Silva et al., ${ }^{34}$ ao analisar os dados da Pesquisa de Orçamento Familiar 2002-2003, encontraram excesso de peso em idosos de alta renda apesar de, supostamente, terem mais acesso a serviços de saúde e hábitos de vida saudáveis.

Tanto ex-fumantes quanto fumantes atuais apresentaram associação inversa com o excesso de peso, corroborando estudos prévios, ${ }^{5,35}$ possivelmente explicada pelo maior gasto metabólico apresentado por fumantes em relação às outras categorias. Achados demostram que quanto maior a quantidade de cigarros fumados, menor é o IMC, e neste processo a nicotina parece ter papel fundamental, pois atua no controle e regulação do apetite e da saciedade. ${ }^{36}$

O baixo peso foi associado aos idosos sem companheira/o, contradizendo achados de estudos prévios. ${ }^{4,30}$ Para o sexo masculino, estar sem companheira tem um impacto negativo na vida, principalmente nesse grupo etário, no qual as mulheres costumam mostrar-se como cuidadoras na relação. ${ }^{37} \mathrm{~A}$ falta de companheira pode levar ao isolamento social, falta de autocuidado e, conforme observado pelo estudo, maior probabilidade de ter baixo peso. ${ }^{38}$ Segundo uma coorte americana com mil indivíduos, idosos com altos níveis de isolamento social apresentaram declínio motor $80 \%$ mais rápido, comparados aos que não se sentem sozinhos. Da mesma forma, o declínio da atividade social pode levar à redução da capacidade física e mudanças de hábitos alimentares, como a perda de apetite e emagrecimento. ${ }^{8}$

No que se refere à Internet, o não uso associou-se ao baixo peso. Isso pode estar relacionado à falta de contato social e à rede de apoio desses idosos, ao não estarem conectados a esse meio de comunicação. ${ }^{8,39}$ Além disso, o uso de tecnologias tem contribuído para o bem-estar dos idosos, ao conferir maior capacitação, autonomia e prazer a eles. ${ }^{39}$ Tem se revelado importante fator para a redução da prevalência de dependência funcional moderada/grave ${ }^{23}$ declínio cognitivo, melhora dos hábitos de vida, como a da prática de atividade física, de hábitos alimentares e tabágicos. ${ }^{40}$

Em relação à capacidade funcional, a presença de incapacidade leve/moderada foi associada ao excesso de peso, efeito observado em outros estudos..$^{10,13,41}$ Sabe-se que o envelhecimento predispõe alterações na composição corporal, que podem comprometer a força e predispor a fraqueza e/ou fragilidade, tornando os idosos mais incapazes. ${ }^{7} \mathrm{O}$ excesso de peso também está associado ao maior desenvolvimento de comorbidades, perda de mobilidade, incapacidade e pior qualidade de vida.,10

Homens com excesso de peso apresentaram menor chance de perceber seu estado de saúde de forma ruim/muito ruim, diferentemente do observado por Arnold et al., ${ }^{13}$ para quem a perda de peso e o ganho/perda intermitente de peso foram associados à pior autopercepção do estado 
de saúde. O excesso de peso é conhecido fator de risco para depressão em idosos; ${ }^{11}$ além disso, o sedentarismo e a presença de multimorbidades, comuns entre aqueles que apresentam excesso de peso, podem deteriorar a qualidade de vida, aumentar a probabilidade de apresentar incapacidade e agravar a ruim autopercepção do estado de saúde.,16

Entre as limitações do estudo, podemos citar o delineamento transversal e as informações autorreferidas. Embora não seja possível estabelecer relação causal, as associações observadas têm sustentação da literatura. As informações autorreferidas, assim como o uso de Proxy respondente, podem repercutir na interpretação errônea da informação pelo entrevistado e/ou omissão de respostas legítimas quando o idoso recebeu auxílio do cuidador/acompanhante. Contudo, salientase que a utilização de instrumentos validados e o treinamento extenso da equipe de campo foram realizados para reduzir vieses.

Como ponto forte do estudo, destaca-se a avaliação do estado nutricional, assim como a aplicação do questionário, realizada por meio de instrumentos validados e procedimentos usados em estudos de diferentes populações de idosos. Além disso, a pesquisa envolveu dados de base populacional, com amostra representativa da população idosa do município de Florianópolis, SC.

\section{Conclusão}

Ser insuficientemente ativo no lazer, relatar três ou mais morbidades e apresentar incapacidade leve e moderada/grave nas AVD foram associados ao excesso de peso em mulheres idosas do município de Florianópolis. No que se refere aos homens, viver sem companheiro, menor escolaridade e não usar Internet, ao baixo peso, enquanto a renda (primeiro e segundo quartil) e apresentar incapacidade moderada/grave nas AVD, ao excesso de peso. Ser ex-fumante, fumante atual e relatar ruim/muito ruim autopercepção do estado de saúde apresentaram associação inversa com o excesso de peso. Ainda nos homens, ter 80 anos ou mais e renda no terceiro quartil foi associado a ambos, baixo peso e excesso de peso.

Estes achados permitirão desenvolver estratégias para a prevenção e promoção da saúde, com intuito de que os idosos mantenham, por maior tempo, a independência, autonomia e qualidade de vida.

\section{Referências}

1. Gontijo S. Envelhecimento ativo: uma política de saúde. Envelhecimento ativo: uma política de saúde. Brasília: Organização Pan-Americana da Saúde; 2005.

2. Organização Pan-Americana da Saúde. Doenças crónico-degenerativas e obesidade: estratégia mundial sobre alimentação saudável, actividade fisíca e saúde. Brasília: OPAS; 2003. 
3. Brownie S. Why are elderly individuals at risk of nutritional deficiency? International Journal of Nursing Practice 2006; 12(2):110-8.

4. Campos MAG, Pedroso ERP, Lamounier JA, Colosimo EA, Abrantes MM. Estado nutricional e fatores associados em idosos. Rev Assoc Med Bras. 2006; 52(4):214-21.

5. Coqueiro RDS, Barbosa AR, Borgatto AF. Nutritional status, health conditions and sociodemographic factors in the elderly of Havana, Cuba: data from SABE survey. J Nutr Health Aging. 2010; 14(10):803-8.

6. Lisko I, Stenholm S, Raitanen J, Hurme M, Hervonen A, Jylhä M, et al. Association of body mass index and waist circumference with physical functioning: The vitality 90+ study. J Gerontol A Biol Sci Med Sci 2015; 70(7):885-91.

7. Barbosa AR, Souza JMP, Lebrão ML, Marucci MdFN. Estado nutricional e desempenho motor de idosos de São Paulo. Rev Assoc Med Bras. 2007; 53(1):75-9.

8. Buchman AS, Boyle PA, Wilson RS, James BD, Leurgans SE, Arnold SE, et al. Loneliness and the rate of motor decline in old age: the rush memory and aging project, a community-based cohort study. BMC Geriatrics 2010; 10(1):77.

9. Samper-Ternent R, Al Snih S. Obesity in older adults: epidemiology and implications for disability and disease. Reviews in Clinical Gerontology 2012; 22(1):10-34.

10. Jensen GL, Hsiao PY. Obesity in older adults: relationship to functional limitation. Curr Opin Clin Nutr Care 2010; 13(1):46-51.

11. Xiang X, An R. Obesity and onset of depression among U.S. middle-aged and older adults. J Psychosom Res. 2015; 78(3):242-8.

12. Barreto SM, Passos V, Lima-Costa MFF. Obesity and underweight among Brazilian elderly: the Bambuí Health and Aging Study. Cad. Saúde Pública 2003; 19(2):605-12.

13. Arnold AM, Newman AB, Cushman M, Ding J, Kritchevsky S. Body weight dynamics and their association with physical function and mortality in older adults: the cardiovascular health study. J Gerontol A Biol Sci Med Sci. 2010; 65(1):63-70.

14. Boscatto EC, Duarte MdF, Coqueiro RdS, Barbosa AR. Nutritional status in the oldest elderly and associated factors. Rev Assoc Med Bras. 2013; 59(1):40-7.

15. Nascimento CdM, Ribeiro AQ, Cotta RMM, Acurcio FdA, Peixoto SV, Priore SE, et al. Nutritional status and associated factors among the elderly in Viçosa, Minas Gerais State, Brazil. Cad. Saúde Pública 2011; 27(12):2409-18.

16. Friedmann JM, Elasy T, Jensen GL. The relationship between body mass index and self-reported functional limitation among older adults: a gender difference. J Am Geriatr Soc. 2001; 49(4):398-403.

17. Giehl MWC, Schneider IJC, Corseuil HX, Benedetti TRB, d'Orsi, E. Physical activity and environment perception among older adults: a population study in Florianópolis, Brazil. Rev Saúde Pública 2012; 46(3):516-25. .

18. Chumlea WC, Guo S, Roche A, Steinbaugh M. Prediction of body weight for the nonambulatory elderly from anthropometry. J Am Diet Assoc. 1988; 88(5):564-68. 
19. American Academy of Family Physicians. American Dietetic Association. National Council on the Aging. Nutrition screening e intervention resources for healthcare professionals working with older adults. Nutrition Screening Initiative. Washington: American Dietetic Association; 2002

20. Brasil, Ministério da Saúde. Protocolos do Sistema de Vigilância Alimentar e Nutricional (SISVAN) na assistência à saúde. Brasília: Ministério da Saúde; 2008.

21. Lima CT, Freire ACC, Silva APB, Teixeira RM, Farrell M, Prince M. Concurrent and construct validity of the AUDIT in an urban Brazilian sample. Alcohol and Alcoholism 2005; 40(6):584-9.

22. Craig CL, Marshall AL, Sjostrom M, Bauman AE, Booth ML, Ainsworth BE, et al. International physical activity questionnaire: 12-country reliability and validity. Med Sci Sports Exerc. 2003; 35(8):1381-95.

23. Medeiros FdL, Xavier AJ, Schneider IJC, Ramos LR, Sigulem D, d'Orsi E. Inclusão digital e capacidade funcional de idosos residentes em Florianópolis, Santa Catarina, Brasil (EpiFloripa 2009-2010). Rev Bras Epidemiol. 2012; 15(1):106-22.

24. Instituto Brasileiro de Geografia e Estatística. Instrumento de Coleta. Pesquisa nacional por amostra de domicílios: PNAD [Internet]. [acesso em: 20 mar. 2009]. Disponível em: http://www.ibge.gov.br/ home/estatistica/populacao/trabalhoerendimento/pnad2003/coeficiente_brasil.shtm

25. Rosa TEC, Benício MHDA, Dias MR. Fatores determinantes da capacidade funcional entre idosos. Rev. Saúde Pública 2003; 37(1):40-8.

26. Ware Jr JE, Kosinski M, Keller SD. A 12-item short-form health survey: construction of scales and preliminary tests of reliability and validity. Medical Care 1996; 34(3):220-33.

27. Mendonça CP, Anjos LA. Aspectos das práticas alimentares e da atividade física como determinantes do crescimento do sobrepeso/obesidade no Brasil Dietary and physical activity factors as determinants of the increase. Cad Saúde Pública 2004; 20(3):698-709.

28. Villareal DT, Apovian CM, Kushner RF, Klein S. Obesity in older adults: technical review and position statement of the American Society for Nutrition and NAASO, The Obesity Society. Obesity Research 2005; 13(11):1849-63.

29. Svendsen OL, Hassager C, Christiansen C. Age-and menopause-associated variations in body composition and fat distribution in healthy women as measured by dual-energy X-ray absorptiometry. Metabolism 1995; 44(3):369-73.

30. Meneguci J, Santos ÁdS, Damião R. Avaliação Nutricional e fatores sociodemográficos, condições de saúde e hábitos associados em idosos. O Mundo da Saúde 2014; 38(2):277-85.

31. Machado VSS, Valadares ALR, Costa-Paiva LH, Osis MJ, Sousa MH, Pinto-Neto AM. Aging, obesity, and multimorbidity in women 50 years or older: a population-based study. Menopause 2013; 20(8):818-24.

32. Guh DP, Zhang W, Bansback N, Amarsi Z, Birmingham CL, Anis AH. The incidence of comorbidities related to obesity and overweight: a systematic review and meta-analysis. BMC Public Health 2009; 9(1):88.

33. Monteiro CA, Conde WL, Popkin BM. Independent effects of income and education on the risk of obesity in the Brazilian adult population. J Nutr. 2001; 131(3):881S-886S. 
34. Silva VS, Souza I, Petroski EL, Silva DAS. Prevalência e fatores associados ao excesso de peso em idosos brasileiros. Revista Brasileira de Atividade Física \& Saúde 2011; 16(4):289-94.

35. Silveira EA, Kac G, Barbosa LS. Prevalência e fatores associados à obesidade em idosos residentes em Pelotas, Rio Grande do Sul, Brasil: classificação da obesidade segundo dois pontos de corte do índice de massa corporal. Cad. Saúde Pública 2009; 25(7):1569-77.

36. Chatkin R, Chatkin JM. Smoking and changes in body weight: can physiopathology and genetics explain this association? J Bras Pneumol. 2007; 33(6):712-9.

37. Zaninotto P, Falaschetti E, Sacker A. Age trajectories of quality of life among older adults: results from the english longitudinal study of ageing. Qual Life Res. 2009; 18(10):1301-09.

38. Olayiwola IO, Ketiku AO. Socio-demographic and nutritional assessment of the elderly Yorubas in Nigeria. Asia Pac J Clin Nutr 2006; 15(1):95-101.

39. Tampubolon G. Delineating the third age: joint models of older people's quality of life and attrition in Britain 2002-2010. Aging Ment Health 2015; 19(7):576-83.

40. Xavier AJ, d'Orsi E, Wardle J, Demakakos P, Smith SG, von Wagner C. Internet use and cancerpreventive behaviors in older adults: findings from a longitudinal cohort study. Cancer Epidemiol Biomarkers Prev. 2013; 22(11):2066-74.

41. Corona LP, Pereira de Brito TR, Nunes DP, Alexandre TS, Santos JLF, Duarte YA, et al. Nutritional status and risk for disability in instrumental activities of daily living in older Brazilians. Public Health Nutr. 2014; 17(2):390-95.

Recebido: 20/4/2016

Aceito: 15/8/2016 